\title{
Stability of higher order singular points of Poisson manifolds and Lie algebroids
}

\author{
Jean-Paul Dufour * \\ Département de Mathématiques \\ CNRS-UMR 5030, Université Montpellier 2 \\ Aissa Wade ${ }^{\dagger \ddagger}$ \\ Department of Mathematics, Penn State University \\ University Park, PA 16802
}

\begin{abstract}
We study the stability of singular points for smooth Poisson structures as well as general Lie algebroids. We give sufficient conditions for stability lying on the first-order approximation (not necessarily linear) of the given Poisson structure or Lie algebroid at a singular point. The main tools used here are the classical Lichnerowicz-Poisson cohomology and the deformation cohomology for Lie algebroids recently introduced by Crainic and Moerdijk. We also provide several examples of stable singular points of order $k \geq 1$ for Poisson structures and Lie algebroids. Finally, we apply our results to pre-symplectic leaves of Dirac manifolds.
\end{abstract}

Keywords: Poisson structure, Lie algebroid, Lichnerowicz-Poisson cohomology, deformation cohomology, stable points.

2000 Mathematics Subject Classification: 53D17, 34Dxx, 37C15.

*email: dufourj@math.univ-montp2.fr

†email: wade@math.psu.edu

${ }^{\ddagger}$ Research partially supported by the Shapiro Funds 


\section{Introduction and main results}

Every bivector field on a smooth finite-dimensional manifold $M$ will be considered as a map $\Lambda: M \rightarrow \bigwedge^{2} T M$. We endow the set $\mathcal{V}_{2}(M)$ of all smooth bivector fields with the $\mathcal{C}^{s}$-topology, i.e. the topology of uniform convergence on compact sets for $\mathcal{C}^{s}$-differentiable maps and their derivatives up to order $s$. The integer $s$ will be made more precise later on.

A smooth Poisson structure on $M$ is a bivector field $\pi$ that satisfies the equation $[\pi, \pi]=0$, where $[$,$] is the Schouten-Nijenhuis bracket on multi-$ vector fields (see [K85]). A singular point of $\pi$ is a point $m \in M$ satisfying $\pi(m)=0$. Such a point $m$ is said to be stable if every Poisson structure $\tilde{\pi}$, which is close enough to $\pi$, has a singular point near $m$.

The following result, due to M. Crainic and R.-L. Fernandès, was announced at the 4th international conference on Poisson geometry (held in Luxembourg, June 2004):

Theorem 1.1 ([CF04] Let $\mathfrak{g}$ be the Lie algebra corresponding to the 1-jet of $\pi$ at a singular point $m_{0}$. If the second scalar cohomology group of $\mathfrak{g}$ vanishes then $m_{0}$ is stable. Conversely, suppose $\mathfrak{g}$ is a Lie algebra such that, every singular point of a degenerate Poisson structure whose 1-jet at that point corresponds to $\mathfrak{g}$ is stable, then $H^{2}(\mathfrak{g}, \mathbb{R})=\{0\}$.

After a quick reading of this result, one may think that $H^{2}(\mathfrak{g}, \mathbb{R})=\{0\}$ is a necessary and sufficient condition for the stability of a singular point of a Poisson structure. However, a curious phenomenon occurs: there are plenty of stable singular points for Poisson structures having a zero 1-jet, hence $H^{2}(\mathfrak{g}, \mathbb{R}) \neq 0$. In Section 2 we will establish a stability criterion for general singularities which improves the one given by Crainic and Fernandès.

First, we recall some definitions. Two smooth maps from $M$ to a manifold $N$ have the same $k$-jet at a point $m \in M$ if they coincide at $m$ and the derivatives of their local expressions, relative to any local coordinate system, agree at that point up to order $k(k \geq 0)$. We denote by $J_{\Phi}^{k}(m)$ the $k$-jet at $m$ of a function $\Phi \in C^{\infty}(M, N)$. Precisely, $J_{\Phi}^{k}(m)$ is the equivalence class of $\Phi$ under the equivalence relation "to have the same $k$-jet at $m$ ".

A singularity of order $k$ for a Poisson structure $\pi$ on $M$ is a point $m \in M$, where the $(k-1)$-jet of $\pi$ vanishes but not its $k$-jet $(k>0)$. We say that such a singularity is $k$-stable if, for every neighborhood $\Omega$ of $m$ in $M$, there is a neighborhood $\mathcal{W}$ of $\pi$ in $\mathcal{V}_{2}(M)$ such that every Poisson structure in $\mathcal{W}$ has a singularity of order $k$ in $\Omega$. 
Associated to any singular point $m$ of order $k$ for a Poisson structure $\pi$, there is a $k$-homogeneous Poisson structure on $T_{m} M$ which is determined, up to isomorphism, by the $k$ th-order terms of the Taylor expansion of $\pi$ at $m$. This new Poisson tensor $\pi^{(k)}$ will be called the $k$-homogeneous part of $\pi$ at $m$. We associate to $\pi^{(k)}$ the homogeneous Lichnerowicz-Poisson cohomology complex (see [DZ04]):

$$
\mathcal{V}_{1}^{(s-k+1)}\left(T_{m} M\right) \quad \stackrel{\partial_{1}^{s-k+1}}{\longrightarrow} \mathcal{V}_{2}^{(s)}\left(T_{m} M\right) \quad \stackrel{\partial_{2}^{s}}{\longrightarrow} \mathcal{V}_{3}^{(s+k-1)}\left(T_{m} M\right) \cdots
$$

where $\mathcal{V}_{r}^{(s)}\left(T_{m} M\right)$ is the space of $s$-homogeneous $r$-vector fields on $T_{m} M$ (with $\mathcal{V}_{r}^{(s)}\left(T_{m} M\right)=\{0\}$ for $s<0$ ), and the operators $\partial_{r}^{\ell}$ are defined by

$$
\partial_{r}^{\ell}(A)=\left[\pi^{(k)}, A\right]
$$

for all $\ell$-homogeneous multi-vector field $A$. The corresponding second cohomology group is

$$
H_{\mathrm{LP}}^{2, s}\left(\pi^{(k)}\right)=\frac{\operatorname{Ker}\left(\partial_{2}^{s}\right)}{\operatorname{Im}\left(\partial_{1}^{s-k+1}\right)} .
$$

Under these notations, we have the following theorem, where $\mathcal{V}_{2}(M)$ is equipped with the $\mathcal{C}^{2 k}$-topology.

Theorem 1.2 Let $m$ be a singularity of order $k$ for a given Poisson structure whose $k$-homogeneous part at $m$ is $\pi^{(k)}$. If $H_{\mathrm{LP}}^{2, s}\left(\pi^{(k)}\right)=\{0\}$, for any $s=0, \ldots, k-1$, then $m$ is a k-stable singularity.

Our second main result concerns the stability problem for general Lie algebroids. A Lie algebroid $([\mathrm{CW} 99])$ is a triple $(F,[],, \varrho)$ formed by a vector bundle $F$ over a smooth manifold $M$ together with a Lie bracket on the space $\Gamma(F)$ of smooth sections of $F$, and a bundle map $\varrho: F \rightarrow T M$ called the anchor map such that

$$
[X, f Y]=f[X, Y]+\varrho(X) \cdot f Y, \quad \forall X, Y \in \Gamma(F), f \in C^{\infty}(M) .
$$

The distribution Im $\varrho$ induces a singular foliation on $M$ which is called the foliation of the Lie algebroid. A singular point for a Lie algebroid is, by definition, a point $m$ of the base manifold $M$ where the leaf of the associated foliation reduces to that point.

Our goal is to give a criterion for the stability of these singularities. But first, we have to put a suitable topology on the set of Lie algebroid structures 
on a given vector bundle. To do so and to present our results, we begin by recalling that there is a one-to-one correspondence between Lie algebroids and some special Poisson structures.

A fiber-wise linear Poisson structure on a vector bundle $p: E \rightarrow M$ is a Poisson structure on $E$ which has the following properties:

- The bracket of two fiber-wise linear functions is fiber-wise linear.

- The bracket of a fiber-wise linear function and a basic one is basic (every basic function on $E$ has the form $f \circ p$, where $f$ is a function on $M$ ).

- The bracket of two basic functions vanishes.

A fiber-wise linear Poisson structure $\Pi$ has the local form

$$
\Pi=\sum_{1 \leq i<j \leq r} f_{i, j}^{s}(x) y_{s} \partial y_{i} \wedge \partial y_{j}+\sum_{1 \leq i \leq r} \sum_{1 \leq j \leq d} g_{i, j}(x) \partial y_{i} \wedge \partial x_{j}
$$

in any system of fibered coordinates $(x, y)$, where $x=\left(x_{1}, \ldots, x_{d}\right)$ is a coordinate system on the base manifold $M$ and $y=\left(y_{1}, \ldots, y_{r}\right)$ consists of functions that are linear on the fibers $\left(\partial z\right.$ stands for $\left.\frac{\partial}{\partial z}\right)$.

It is well-known that there is a one-to-one correspondence between Lie algebroid structures on a vector bundle $F$ and fiber-wise linear Poisson structures on the total space of its dual $E=F^{*}$ (see for instance [CW99]). Hereafter, we identify these two concepts: the concept of a Lie algebroid will be sometimes employed instead of that of a fiber-wise linear Poisson structure. In particular, the topology we put on the set of Lie algebroid structures on a given vector bundle $F$ is the one which corresponds to the $\mathcal{C}^{s}$-topology for bivector fields on the total space of the dual vector bundle $E=F^{*}$ (the integer $s$ will be specified later on). A point $m$ of the base manifold $M$ is a singular point of a Lie algebroid if and only if $0_{m}$, the origin of the fiber over $m$, is a singular point of the associated fiber-wise linear Poisson structure $\Pi$. In the above local expression of $\pi$, such a singular point corresponds to a point $x$ with $g_{i, j}(x)=0$.

It makes sense to speak of singularities of order $k$ for a Lie algebroid. By definition, these are singular points $m$ such that $0_{m}$ is a singularity of order $k$ for $\Pi$. The first non-zero terms in the Taylor expansion of $\Pi$ at $0_{m}$ have the local form

$$
\Pi^{(k)}=\sum f_{i, j}^{(k-1), \ell}(x) y_{\ell} \partial y_{i} \wedge \partial y_{j}+\sum g_{i, j}^{(k)}(x) \partial y_{i} \wedge \partial x_{j}
$$

where $g_{i, j}^{(k)}$ (resp. $\left.f_{i, j}^{(k-1), \ell}\right)$ are $k$-homogeneous (resp. $(k-1)$-homogeneous) polynomials. When $k=1$ we have

$$
\Pi^{(1)}=\sum a_{i, j}^{\ell} y_{\ell} \partial y_{i} \wedge \partial y_{j}+\sum b_{i, j}^{\ell} x_{\ell} \partial y_{i} \wedge \partial x_{j}
$$


where $a_{i, j}^{\ell}$ are structure constants for a Lie algebra $\mathfrak{g}$ and $b_{i, j}^{\ell}$ are constants. It corresponds to the algebroid associated to a linear action of the Lie algebra $\mathfrak{g}$ on the tangent space of the base manifold at a given point (see for example [CW99]).

As for general Poisson structures, the first homogeneous terms of the Taylor expansion at $0_{m}$, denoted $\Pi^{(k)}$, determine a $k$-homogeneous Lie algebroid. Moreover, we can see that $\Pi^{(k)}$ is a fiber-wise linear Poisson structure on $V=T_{0_{m}} E$, viewed as a trivial vector bundle with base $T_{m} M$ and fiber $T_{0_{m}}\left(p^{-1}(m)\right) \simeq p^{-1}(m)$. We say that the algebroid singularity $m$ is $k$-stable if, for every neighborhood $\Omega$ of $m$ in $M$, there is a neighborhood $\mathcal{W}$ of $\Pi$ in $\mathcal{V}_{2}(E)$ such that every fiber-wise linear Poisson structure in $\mathcal{W}$ has a singularity of order $k$ in $\Omega$. Even though Lie algebroids can be viewed as specific Poisson structures, the Lichnerowicz-Poisson cohomology used in Theorem 1.2 is not suitable for the study of the stability problem for Lie algebroids. One needs to use the deformation cohomology introduced recently by Crainic and Moerdijk (see [CM04]). This is also called the linear fiber-wise Poisson cohomology for Lie algebroids (see [DZ04]). Hereafter, we will use a local and homogeneous version. We attach to $\Pi^{(k)}$ the deformation complex

$$
\mathcal{V}_{1, \text { lin }}^{(s-k+1)}\left(T_{0_{m}} E\right) \quad \stackrel{\partial_{1}^{s-k+1}}{\longrightarrow} \quad \mathcal{V}_{2, l i n}^{(s)}\left(T_{0_{m}} E\right) \quad \stackrel{\partial_{2}^{s}}{\longrightarrow} \mathcal{V}_{3, \text { lin }}^{(s+k-1)}\left(T_{0_{m}} E\right) \cdots
$$

which is by definition the sub-complex of the Lichnerowicz-Poisson complex. Precisely, we replace $\mathcal{V}_{r}^{(s)}\left(T_{0_{m}} E\right)$ by its sub-space $\mathcal{V}_{r, \text { lin }}^{(s)}\left(T_{0_{m}} E\right)$ formed by $s$ homogeneous $r$-vector fields $K$ which satisfies the following properties:

- $K\left(d f_{1} \ldots, d f_{r}\right)$ is fiber-wise linear if $f_{1}, \ldots, f_{r}$, are fiber-wise linear, - $K\left(d f_{1} \ldots, d f_{r}\right)$ is basic if $f_{1}, \ldots, f_{r-1}$, are fiber-wise linear and $f_{r}$ is basic, - $K\left(d f_{1} \ldots, d f_{r}\right)=0$ if at least two of the $f_{i}$ are basic.

In any local coordinates $(x, y)$ chosen as above, elements of $\mathcal{V}_{r, l i n}^{(s)}(V)$ have the form

$\sum f_{i_{1} \ldots i_{r}}^{(s-1), u}(x) y_{u} \partial y_{i_{1}} \wedge \cdots \wedge \partial y_{i_{r}}+\sum g_{j_{1} \ldots j_{r-1}, t}^{(s)}(x) \partial x_{t} \wedge \partial y_{j_{1}} \wedge \cdots \wedge \partial y_{j_{r-1}}$

where $g_{j_{1} \ldots j_{r-1}, t}^{(s)}$ are $s$-homogeneous polynomials and $f_{i_{1} \ldots i_{r}}^{(s-1), u}$ are $(s-1)$ homogeneous polynomials on $T_{m} M$. Denote by $H_{\text {lin }}^{2, s}\left(\Pi^{(k)}\right)$ the second group of cohomology for the above cohomology complex.

Under these notations we have the following theorem, where $\mathcal{V}_{2}(E)$ is equipped with the $\mathcal{C}^{2 k}$-topology. 
Theorem 1.3 Let $m$ be a singularity of order $k$ for the algebroid $\Pi$ and $\Pi^{(k)}$ its $k$-homogeneous part at $m$. If $H_{\text {lin }}^{2, s}\left(\Pi^{(k)}\right)=\{0\}$, for any $s=0, \ldots, k-1$, then $m$ is a $k$-stable algebroid singularity.

Section 2 contains the proofs of our main results (Theorems 1.2 and 1.3). In Section 3. we will give examples and shows why most of the singularities of higher order are stable (at least in dimension 3). Section 4 gives a method for generalizing the above results to singular leaves (not necessarily reduced to a point).

\section{Proofs of the main theorems}

We will start by establishing Theorem 1.2 First, one can notice the following

Remark 2.1 a) The hypothesis of Theorem 1.2 says that $\partial_{2}^{s}$ are one-to-one for $s=0, \ldots, k-2$, and

$$
\operatorname{Ker} \partial_{2}^{k-1}=\operatorname{Im} \partial_{1}^{0}
$$

b) In contrast with Theorem 1.1, we do not have a "kind of" converse to Theorem 1.2 when $k>1$. A counter-example is given by the Poisson structure $\pi^{(2)}=x y \frac{\partial}{\partial x} \wedge \frac{\partial}{\partial y}$ on $\mathbb{R}^{2}$. Every singular point $m$ of order 2 for a Poisson structure $\pi$ whose 2-jet at that point is isomorphic to $\pi^{(2)}$ is 2stable. Observe that $\pi$ itself is (locally) isomorphic to $\pi^{(2)}$. However the Poisson 2-cocycle $\frac{\partial}{\partial x} \wedge \frac{\partial}{\partial y}$ is not a coboundary. Hence, $H_{L P}^{2,0}\left(\pi^{(2)}\right) \neq\{0\}$.

Given a neighborhood $\Omega$ of $m$ in $M$, we choose a local coordinate system $\left(x_{1}, \ldots, x_{n}\right)$, defined on an open neighborhood $U$ of $m$ that is contained $\Omega$. For any bivector field $\Lambda$ on $M$ and for any point $p$ in $U$, we denote by $\Lambda_{p}^{(s)}$ the $s$-homogeneous terms of the Taylor expansion of $\Lambda$ at $p$ in the above coordinates. We can view $\Lambda_{p}^{(s)}$ as an element of $\mathcal{V}_{2}^{(s)}\left(\mathbb{R}^{n}\right)$, then the $(k-1)$-jet extension of $\Lambda$ is given by:

$$
\begin{aligned}
\mathcal{J}_{\Lambda}^{k-1}: & U \longrightarrow \mathcal{V}_{2}^{(0)}\left(\mathbb{R}^{n}\right) \times \cdots \times \mathcal{V}_{2}^{(k-1)}\left(\mathbb{R}^{n}\right) \\
p & \longmapsto\left(\Lambda_{p}^{(0)}, \ldots, \Lambda_{p}^{(k-1)}\right) .
\end{aligned}
$$

Accordingly $\pi^{(k)}=\pi_{m}^{(k)}$ may be thought of as an element of $\mathcal{V}_{2}^{(k)}\left(\mathbb{R}^{n}\right)$ and the associated linear map $\partial_{1}^{0}$ sends $\mathcal{V}_{1}^{(0)}\left(\mathbb{R}^{n}\right)$ into $\mathcal{V}_{2}^{(k-1)}\left(\mathbb{R}^{n}\right)$. From now on, 
we will use the notation

$$
A=\{0\} \times \cdots \times\{0\} \times \operatorname{Im}\left(\partial_{1}^{0}\right)
$$

which is a subspace of $\mathcal{V}_{2}^{(0)}\left(\mathbb{R}^{n}\right) \times \cdots \times \mathcal{V}_{2}^{(k-1)}\left(\mathbb{R}^{n}\right)$. Then, one gets

Lemma 2.2 The image of the differential of $\mathcal{J}_{\pi}^{k-1}$ at $m$ coincides with A.

The proof of this lemma is straightforward. It is left to the reader.

Given a point $p$ in $U$ and bivector field $\Lambda$ on $M$, we define

$$
\mathcal{F}_{p, \Lambda}: \mathcal{V}_{2}^{(0)}\left(\mathbb{R}^{n}\right) \times \cdots \times \mathcal{V}_{2}^{(k-1)}\left(\mathbb{R}^{n}\right) \longrightarrow \mathcal{V}_{3}^{(k-1)}\left(\mathbb{R}^{n}\right) \times \cdots \times \mathcal{V}_{3}^{(2 k-2)}\left(\mathbb{R}^{n}\right)
$$

such that the components of $\mathcal{F}_{p, \Lambda}\left(v_{0}, \cdots, v_{k-1}\right)$ are given by

$$
\mathcal{F}_{p, \Lambda}^{\ell}\left(v_{0}, \cdots, v_{k-1}\right)=\sum_{i \leq \ell-k}\left[v_{i}, \Lambda_{p}^{(\ell-i)}\right]+\frac{1}{2} \sum_{\ell-k<i, j \leq \ell, i+j=\ell}\left[v_{i}, v_{j}\right],
$$

for all $k \leq \ell \leq 2 k-1$, and where [, ] is the Schouten-Nijenhuis bracket. One has:

Lemma 2.3 Under the hypothesis of Theorem 1.2, $A$ is the kernel of the differential of $\mathcal{F}_{m, \pi}$ at the origin $\mathbf{O}$.

Proof: When $k=1$, one gets $\mathcal{F}_{m, \pi}\left(v_{0}\right)=\left[v_{0}, \pi^{(1)}\right]$. Differentiating, one can easily check that $\operatorname{Ker}\left(\left(\mathcal{F}_{m, \pi}\right)_{*}(\mathbf{O})\right)=\operatorname{Ker}\left(\partial_{2}^{0}\right)=\operatorname{Im}\left(\partial_{1}^{0}\right)$. Now, assume that $k>1$, then the equation

$$
\left(\left(\mathcal{F}_{m, \pi}\right)_{*}(\mathbf{O})\right)\left(w_{0}, \ldots, w_{k-1}\right)=0
$$

gives the system of equations

$$
\begin{gathered}
{\left[w_{0}, \pi^{(k)}\right]=0, \quad\left[w_{1}, \pi^{(k)}\right]+\left[w_{0}, \pi^{(k+1)}\right]=0, \ldots,} \\
{\left[w_{k-1}, \pi^{(k)}\right]+\cdots+\left[w_{0}, \pi^{(2 k-1)}\right]=0}
\end{gathered}
$$

The first equation of this system says that $w_{0} \in \operatorname{ker}\left(\partial_{2}^{0}\right)$. By Remark 2.1] one gets $w_{0} \in \operatorname{Im}\left(\partial_{1}^{1-k}\right)$. Using the fact that $\operatorname{Im}\left(\partial_{1}^{1-k}\right)=\{0\}$ for $k>1$, one obtains $w_{0}=0$. By an iteration procedure, one gets

$$
w_{0}=\cdots=w_{k-2}=0 \quad \text { and } \quad \partial_{2}^{k-1}\left(w_{k-1}\right)=0, \quad \forall k>1 .
$$

This shows that the kernel of the differential of $\mathcal{F}_{m, \pi}$ at $\mathbf{O}$ is exactly $A$. 


\section{Proof of Theorem 1.2;}

Once for all, we fix a complement subspace $B$ of $A$, i.e.

$$
\mathcal{V}_{2}^{(0)}\left(\mathbb{R}^{n}\right) \times \cdots \times \mathcal{V}_{2}^{(k-1)}\left(\mathbb{R}^{n}\right)=A \oplus B
$$

Lemma 2.3 implies that the differential of $\mathcal{F}_{m, \pi}$ at the origin is one-to-one, when restricted to $B$. Applying the implicit function theorem, one gets that $\mathcal{F}_{m, \pi}$ is an injective immersion when it is restricted to a neighborhood $\mathcal{B}_{0}$ of the origin in $B$. Note that $\mathcal{F}_{p, \Lambda}$ depends continuously on $\Lambda$ in the $\mathcal{C}^{2 k}$ topology. A classical singularity technique (see Lemma A, p. 61, GG73]) implies that there is a neighborhood $\mathcal{B}_{1} \subset \mathcal{B}_{0}$ of the origin in $B$, a neighborhood $\Omega_{1} \subset U$ of $m$, and a neighborhood $\mathcal{W}_{1}$ of $\pi$ in $\mathcal{V}_{2}(M)$ such that, for every $p$ in $\Omega_{1}$ and for every $\Lambda$ in $\mathcal{W}_{1}, \mathcal{F}_{p, \Lambda}$ is one-to-one when restricted to $\mathcal{B}_{1}$.

It follows from Lemma 2.2 that the map $\mathcal{J}_{\pi}^{k-1}$ is transversal to $B$ at the origin. By transversality, there is a neighborhood $\mathcal{W}_{2}$ of $\pi$ in $\mathcal{V}_{2}(M)$ such that, for every $\Lambda$ in $\mathcal{W}_{2}, \mathcal{J}_{\Lambda}^{k-1}$ intersects (transversally) $\mathcal{B}_{1}$ at a point $p \in \Omega_{1}$. Set $\mathcal{W}=\mathcal{W}_{1} \cap \mathcal{W}_{2}$. Now pick a Poisson structure $\Lambda$ in $\mathcal{W}$. We know that there is a point $p$ in $\Omega_{1}$ where $\mathcal{J}_{\Lambda}^{k-1}(p)$ intersects $\mathcal{B}_{1}$. Considering the equation $[\Lambda, \Lambda]=0$, we can notice that the terms of order $k-1, \ldots, 2 k-2$ in the Taylor expansion of $[\Lambda, \Lambda]$ at that point $p$ give

$$
\mathcal{F}_{p, \Lambda}\left(\mathcal{J}_{\Lambda}^{k-1}(p)\right)=0
$$

The injectivity of $\left.\left(\mathcal{F}_{p, \Lambda}\right)\right|_{\mathcal{B}_{1}}$ implies

$$
\mathcal{J}_{\Lambda}^{k-1}(p)=0 .
$$

If necessary, we may replace $\mathcal{W}$ by a smaller neighborhood of $\pi$ to ensure that the $k$-jet of $\Lambda$ at $p$ is non-trivial. So, $p$ is the singularity point for $\Lambda$ we sought. This completes the proof of Theorem 1.2

Important remark 2.4 In our study there is no need for the singularity to be isolated. In fact, from the proof of Theorem 1.2, one sees that the set of singularities of order $k$ for Poisson structures $\Lambda$ sufficiently close to $\pi$ is (locally) formed by points $p$ such that $\mathcal{J}_{\Lambda}^{k-1}(p) \in \mathcal{B}_{1}$, for each $k$ fixed. So, this set is, by transversality, a sub-manifold of dimension $r=\operatorname{dim}(A)$. One can also notice that this manifold depends continuously on $\Lambda$, in a natural sense. 
The proof of Theorem 1.3 is a direct adaptation of that of Theorem 1.2 Essentially, one replaces the symbol $\mathcal{V}_{u}^{(s)}$ by $\mathcal{V}_{u, \text { lin }}^{(s)}$ and

$$
\begin{aligned}
\mathcal{J}_{\Lambda}^{k-1}: U & \longrightarrow \mathcal{V}_{2}^{(0)}\left(\mathbb{R}^{n}\right) \times \cdots \times \mathcal{V}_{2}^{(k-1)}\left(\mathbb{R}^{n}\right) \\
p & \longmapsto\left(\Lambda_{p}^{(0)}, \ldots, \Lambda_{p}^{(k-1)}\right)
\end{aligned}
$$

by

$$
\begin{aligned}
\mathcal{J}_{\Lambda}^{k-1}: U & \longrightarrow \mathcal{V}_{2, \text { lin }}^{(0)}\left(\mathbb{R}^{d} \times \mathbb{R}^{r}\right) \times \cdots \times \mathcal{V}_{2, \text { lin }}^{(k-1)}\left(\mathbb{R}^{d} \times \mathbb{R}^{r}\right) \\
p & \longmapsto\left(\Lambda_{0_{p}}^{(0)}, \ldots, \Lambda_{0_{p}}^{(k-1)}\right),
\end{aligned}
$$

where $d$ and $r$ are the dimensions of the base manifold and the fiber, respectively.

\section{Examples}

\subsection{Singularities of Poisson structures in dimension 2}

In dimension 2, singularities of Poisson structures are singularities of functions for which there are specific classical techniques (see [M01]). For instance, singularities of topologically stable Poisson structures in Radko's sense (see [R02]) are 1-stable.

\subsection{Singularities of order 1 for Poisson structures}

Let $m$ be a singularity of order 1 for a Poisson structure $\pi$. Denote by $\mathfrak{g}$ the Lie algebra corresponding to the linear part of $\pi$ at $m$. In this case, the hypothesis of Theorem 1.2 reduces to $H_{\mathrm{LP}}^{2,0}\left(\pi^{(1)}\right)=\{0\}$. A straightforward calculation shows that this condition is equivalent to $H^{2}(\mathfrak{g}, \mathbb{R})=0$. This is exactly the condition given by Crainic and Fernandès (see Section 1).

On can notice that if the Lie algebra associated to the linear part of $\pi$ at $m$ is strongly rigid in the sense of Bordemann, Makhlouf, and Petit (see BMP02] then $m$ is 1-stable.

\subsection{Singularities of order 2 for Poisson structures}

It is shown in [DH91] that Poisson structures on a vector space $V$ having the form

$$
\pi^{(2)}=\sum a_{i, j} x_{i} x_{j} \partial x_{i} \wedge \partial x_{j}
$$


are generic among quadratic Poisson structures on $V$. We consider such a Poisson structure $\pi^{(2)}$. It is known that the $a_{i, j}$ are invariants for this Poisson structure (see [DW98]). Set $\lambda_{i}=\sum_{j} a_{i, j}$.

Lemma 3.1 If $\lambda_{i} \neq 0$ for every $i$, and $\lambda_{i}+\lambda_{j} \neq 0$ for $i<j$. then $H_{\mathrm{LP}}^{2, s}\left(\pi^{(2)}\right)=\{0\}$, for $s=0$ and $s=1$.

Note that the hypothesis in the above lemma may occurs only when the dimension is at least 3. The proof of this lemma can be found in M01. This lemma and Theorem 1.2 imply that that singularities of order 2 of quadratic Poisson structures are, in general, stable (in dimension $\geq 3$ ).

\subsection{Singularities of Poisson structures in dimension 3}

Let $\omega$ be a volume form on a 3-dimensional manifold $M$. The map $\pi \mapsto$ $i_{\pi} \omega$ establishes a one-to-one correspondence between Poisson structures and integrable 1-forms (i.e. 1 -forms $\alpha$ such that $\alpha \wedge d \alpha=0$ ). Moreover, $\pi$ and $\alpha=i_{\pi} \omega$ share the same (singular) foliation. In [CN82, Camacho and LinsNeto proved that singularities of order $k$ for these integrable forms are, in general, stable. Hence, the same conclusion holds for Poisson structures in dimension 3 .

In fact, the proof of Theorem 1.2 is inspired by methods used for integrable 1-forms. We will interpret, in terms of Poisson structures, two results for integrable 1-forms obtained by Camacho and Lins-Neto. First, recall that a vector field $X=X_{1} \partial x+X_{2} \partial y+X_{3} \partial z$ on $\mathbb{R}^{3}$, which vanishes at the origin, has an algebraically isolated zero at the origin if the ideal generated by the germs of its components $X_{1}, X_{2}$ and $X_{3}$ has a finite codimension in the space of germs of functions of $x, y$ and $z$ at the origin.

Lemma 3.2 Let $\pi^{(k)}$ a $k$-homogeneous Poisson structure on $\mathbb{R}^{3}$. Suppose its modular vector field $X^{(k-1)}$ with respect to $\omega=d x \wedge d y \wedge d z$ has an algebraically isolated zero at the origin. Then the hypothesis of Theorem 1.2 (i.e $H_{\mathrm{LP}}^{2, s}\left(\pi^{(k)}\right)=\{0\}$, for any $s=0, \ldots, k-1$ ) holds true.

Hence any order $k$ singularity, with $\pi^{(k)}$ as in this lemma, is $k$-stable.

Lemma 3.3 Under the hypothesis of Lemma 3.2 and for all $k>2$, we have

$$
\pi^{(k)}=\frac{1}{k+1} I \wedge X^{(k-1)},
$$

where $I=x \partial x+y \partial y+z \partial z$ is the Liouville vector field. 
This last lemma allows to construct many examples of $k$-stable singularities for $k>2$. For instance, the origin is a $k$-stable singularity for

$\pi=\left(x y^{k-1}-y x^{k-1}\right) \partial x \wedge \partial y+\left(y z^{k-1}-z y^{k-1}\right) \partial y \wedge \partial z+\left(z x^{k-1}-x z^{k-1}\right) \partial z \wedge \partial x$.

\subsection{Singularities of order 1 for Lie algebroids}

We recall that if $m$ is a singularity order 1 for a Lie algebroid then the linear part of this algebroid at that singularity is a Lie algebroid corresponding to a linear action of a Lie algebra $\mathfrak{g}$ on a vector space $V$. We will see that, in this case, the condition of stability of Theorem 1.3 is equivalent to $H^{1}\left(\mathfrak{g}, V^{*}\right)=0$.

Consider a fiber-wise linear Poisson structure that corresponds to an action Lie algebroid $g \ltimes V$. Suppose that it is given by the following expression in a fibered coordinate system $\left(x_{1}, \ldots, x_{d}, y_{1}, \ldots, y_{r}\right)$ :

$$
\Pi^{(1)}=\sum_{k} a_{i, j}^{k} y_{k} \partial y_{i} \wedge \partial y_{j}+\sum_{\ell} b_{p, q}^{\ell} x_{\ell} \partial y_{p} \wedge \partial x_{q},
$$

where $a_{i, j}^{k}$ are structure constants of the Lie algebra $\mathfrak{g}$ and $b_{p, q}^{\ell}$ are constants which determine the action $\mathfrak{g}$ on $V$. Set

$$
\mu=\sum \mu_{u, v} \partial y_{u} \wedge \partial x_{v}
$$

where the $\mu_{u, v}$ are constants. Consider the equation $\left[\Pi^{(1)}, \mu\right]=0$. In this equation, the coefficient of $\partial x_{q} \wedge \partial y_{i} \wedge \partial y_{j}$ gives

$$
\sum_{v}\left(\mu_{i, v} b_{j, q}^{v}-\mu_{j, v} b_{i, q}^{v}\right)-\sum_{u} a_{i, j}^{u} \mu_{u, q}=0 .
$$

But, if $V^{*}$ is identified with the vector space of constant vector fields generated by the vector fields $\partial x_{i}$, then the dual action $\varrho: \mathfrak{g} \rightarrow \operatorname{End}\left(V^{*}\right)$ is given by

$$
\varrho\left(y_{i}\right)\left(\partial x_{j}\right)=-\sum_{v} b_{i, v}^{j} \partial x_{v} .
$$

Furthermore, if we set

$$
\mu_{i}=\sum_{u} \mu_{i, \ell} \partial x_{\ell}
$$

we obtain that Equation (1) can be expressed as

$$
\left\langle\varrho\left(y_{i}\right)\left(\mu_{j}\right)-\varrho\left(y_{j}\right)\left(\mu_{i}\right), d x_{q}\right\rangle=\sum_{u} a_{i, j}^{u} \mu_{u, q} .
$$


More precisely, Equation (1) says that $\mu$ corresponds to a 1-cocycle for the representation $\varrho$. This shows that constant 2-cocycles for the deformation cohomology complex can be identified with 1-cocycles relative to the representation $\varrho$. Similarly, 2-coboundaries for the deformation cohomology complex can be identified with 1-coboundaries relative to the representation $\varrho$. There follows that

$$
H_{\text {lin }}^{2,0}\left(\Pi^{(1)}\right) \cong H^{1}\left(\mathfrak{g}, V^{*}\right) .
$$

In particular, when $\Pi^{(1)}$ is the fiber-wise Poisson structure induced by the natural action of $\mathfrak{g}$ on itself, then $H_{\text {lin }}^{2,0}\left(\Pi^{(1)}\right) \cong H^{1}\left(\mathfrak{g}, \mathfrak{g}^{*}\right)$. As R.-L. Fernandès points out (private communication), one can notice that, in this case, the hypothesis of Theorem 1.3 is stronger than that of Theorem 1.2. More precisely, the natural inclusion $H^{2}(\mathfrak{g}, \mathbb{R}) \hookrightarrow H^{1}\left(\mathfrak{g}, \mathfrak{g}^{*}\right)$ is not onto, in general (since $H^{2}(\mathfrak{g}, \mathbb{R})$ can be viewed as the skew-symmetric part of $H^{1}\left(\mathfrak{g}, \mathfrak{g}^{*}\right)$ ). Consequently, the fact that $m$ is a 1-stable singular point for a Poisson structure whose 1-jet at $m$ corresponds to $\mathfrak{g}$ does not guaranty that it is 1 -stable for the associated Lie algebroid. For instance, if $\mathfrak{g}=\mathfrak{a} \mathfrak{f} \mathfrak{f}(1)$ (i.e. the Lie algebra of affine transformations on $\mathbb{R}^{2}$ ) then the origin $O$ is 1-stable for the associated Lie-Poisson structure on $\mathfrak{g}^{*}$ since $H^{2}(\mathfrak{a f f}(1), \mathbb{R})=\{0\}$. But, the origin is not 1 -stable for the corresponding Lie algebroid.

Furthermore, one can notice that, in the particular case where $\mathfrak{g}$ is reductive and the $X_{i}=\sum b_{i k}^{\ell} x_{\ell} \partial x_{k}$ are diagonal, the Hochschild-Serre factorization theorem (see [HS53] $)$ gives $H_{\text {lin }}^{2,0}\left(\Pi^{(1)}\right)=\{0\} \Longleftrightarrow H^{1}(\mathfrak{g}, \mathbb{R})=\{0\}$.

\subsection{Singularity of order 2 for Lie algebroids}

Consider $\mathbb{R}^{n+1}(n>1)$ with the standard coordinates $\left(x_{0}, x_{1}, \ldots, x_{n}\right)$ and the function $\rho=x_{0}^{2}+\cdots+x_{n}^{2}$. Let $\Pi$ be the fiber-wise linear Poisson structure of on $\mathbb{R}^{2 n+2}$ given by

$$
\Pi^{(2)}=(\rho-1) \sum_{i=0}^{n} \partial x_{i} \wedge \partial y_{i}+\sum_{i<j}\left(x_{i} y_{j}-x_{j} y_{i}\right) \partial y_{i} \wedge \partial y_{j}
$$

This is the Lie algebroid attached to $(\rho-1)$, in the sense of Monnier (see [M02]). Every point lying on the unit sphere is singular. It follows from results proven in M01 that $H_{\text {lin }}^{2, s}\left(\Pi^{(2)}\right)$ vanishes for all $s$ (see Lemma 5.4.3 and Proposition 6.1.2 in M01]). Therefore, the unit sphere is 2-stable. 


\section{Application: stability of non-trivial leaves}

In this section, we will apply our previous results to Dirac manifolds and nontrivial leaves of Lie algebroids. Let $M$ be a smooth $n$-dimensional manifold. We consider the vector bundle $T M \oplus T^{*} M$ together with the Courant bracket defined by

$$
\left[\left(X_{1}, \alpha_{1}\right),\left(X_{2}, \alpha_{2}\right)\right]_{C}=\left(\left[X_{1}, X_{2}\right], \mathcal{L}_{X_{1}} \alpha_{2}-i_{X_{2}} d \alpha_{1}\right),
$$

for any $\left(X_{1}, \alpha_{1}\right),\left(X_{2}, \alpha_{2}\right) \in \Gamma\left(T M \oplus T^{*} M\right)$. Recall that a Dirac structure on $M$ (see [C90]) is a sub-bundle $L \subset T M \oplus T^{*} M$ of rank $n$ which is closed under the Courant bracket and which is isotropic with respect to the bilinear symmetric operation given by

$$
\left\langle\left(X_{1}, \alpha_{1}\right),\left(X_{2}, \alpha_{2}\right)\right\rangle=\frac{1}{2}\left(i_{X_{2}} \alpha_{1}+i_{X_{1}} \alpha_{2}\right),
$$

for any $\left(X_{1}, \alpha_{1}\right),\left(X_{2}, \alpha_{2}\right) \in \Gamma\left(T M \oplus T^{*} M\right)$. In this case, $(M, L)$ is called a Dirac manifold. It is known that every Dirac manifold admits a foliation by pre-symplectic leaves (see C90]). We also know that the dimensions of the leaves have the same parity (see [DW04]).

A pre-symplectic leaf $S$ of $(M, L)$ is singular if every neighborhood of $S$ intersects leaves of higher dimension. We say that the singular leaf $S$ is stable if, for any neighborhood $U$ of $S$, there is a neighborhood $\mathcal{W}$ of $L$ such that all Dirac structures in $\mathcal{W}$ admit a pre-symplectic leaf near $S$ having the same dimension as $S$. Here, we endow the set of Dirac structures with the $C^{s}$-topology by means of the $C^{s}$-topology on local smooth sections of $T M \oplus T^{*} M$ ( $s$ will be specified below).

Let $S$ be a pre-symplectic leaf of $(M, L)$, it is proven in DW04 that if $N \subset M$ is an embedded submanifold that intersects transversally $S$ at $m_{0}$ (in the sense that $\left.T_{m_{0}} M=T_{m_{0}} S \oplus T_{m_{0}} N\right)$ then $L$ induces a Poisson structure on $N$ which vanishes at $m_{0}$. Moreover, this transverse Poisson structure does not depend on $m_{0}$ : it is unique up to Poisson isomorphisms. This is called the transverse Poisson structure along $S$. In the following theorem, we assume that $m_{0}$ is $k$-stable and we endow the set of Dirac structures with the $C^{2 k}$-topology.

Proposition 4.1 Assume that $S$ is an embedded singular pre-symplectic leaf of a Dirac manifold $(M, L)$ and $m_{0} \in S$ is a $k$-stable singular point for the transverse Poisson structure. Then $S$ is stable. 
Proof: Consider a tubular neighborhood of $S$ that corresponds to the vector bundle $p: E \rightarrow S$. Denote by $\pi_{V}$ the transverse Poisson structure defined on an open neighborhood $U \subset E_{m_{0}}$ of $m_{0}$ with $\pi_{V}\left(m_{0}\right)=0$. We set $N=E_{m_{0}}$. Any Dirac structure $L^{\prime}$ sufficiently close to $L_{\mid E}$ has pre-symplectic leaves $S^{\prime}$ near $S$ that intersect $N$ with the transversality relation:

$$
T_{m} E=T_{m} S^{\prime}+T_{m} N
$$

at points $m \in U \subset N$. Using the transversality relation and methods developed in DW04, one can show that $L^{\prime}$ induces a Dirac structure $L_{V}^{\prime}$ in a neighborhood of $m_{0}$ in $N$. Moreover, the fact that $L^{\prime}$ is chosen to be very close to $L_{\mid E}$ implies that $L_{V}^{\prime}$ is the graph of a Poisson structure $\pi_{V}^{\prime}$ which is close to $\pi_{V}$ on $U$ (if necessary, we replace $U$ by a smaller open set). Since $m_{0}$ is $k$-stable for $\pi_{V}$, the Poisson structure $\pi_{V}^{\prime}$ has a singular point of order $k$ in $U$. Obviously, singular points for $\pi_{V}^{\prime}$ are located on leaves of the Dirac manifold $\left(E, L^{\prime}\right)$ that have the same dimension as $S$. In other words, if $m_{1} \in S^{\prime} \cap U$ is a singular point for $\pi_{V}^{\prime}$ then we have a direct sum

$$
T_{m_{1}} E=T_{m_{1}} S^{\prime} \oplus T_{m_{1}} N
$$

moreover $S^{\prime}$ projects diffeomorphically onto $S$ when restricted in a small neighborhood of $m_{0}$ in $M$.

Remark 4.2 In the above proof, $S^{\prime}$ does not globally project onto $S$ via the tubular neighborhood projection, in general (specially, when $S$ is noncompact). For instance, let $M$ be the product of $\mathbb{R}^{2}$ by the torus $\mathbb{T}^{2}$ and suppose that $L_{\pi}$ is the graph of the Poisson structure $\pi$ given by

$$
\pi=y \frac{\partial}{\partial x} \wedge \frac{\partial}{\partial y}+\frac{\partial}{\partial \theta_{1}} \wedge \frac{\partial}{\partial \theta_{2}} .
$$

The submanifolds given by equations $x=$ cte, $y=0$ are singular leaves of $\left(M, L_{\pi}\right)$. By Proposition 4.1, each of these leaves is stable. Note that they are diffeomorphic to $\mathbb{T}^{2}$. Now, we consider

$$
\pi_{\varepsilon}=y \frac{\partial}{\partial x} \wedge \frac{\partial}{\partial y}+\left(\frac{\partial}{\partial \theta_{1}}+\varepsilon \frac{\partial}{\partial x}\right) \wedge \frac{\partial}{\partial \theta_{2}},
$$

where $\varepsilon \in \mathbb{R}$ is a deformation parameter. For $\varepsilon \neq 0$, every 2-dimensional symplectic leaf of $\pi_{\varepsilon}$ is (globally) diffeomorphic to $S^{1} \times \mathbb{R}$. Hence, it is not (globally) diffeomorphic to the torus. 
We should mention that Crainic and Fernandès have obtained (private communication) results on 1-stability of compact leaves of Poisson structures in a stronger sense, i.e. $S$ diffeomorphic to $S^{\prime}$.

Using the notion of transverse Lie algebroid structure along a leaf (see, for instance, [F02]) and the above techniques, one can similarly show the following proposition:

Proposition 4.3 Let $S$ an embedded singular leaf of an algebroid structure. If $m$ is a $k$-stable singularity of the transversal algebroid structure along $S$, then $S$ is stable.

Aknowledgement. We would like to thank Rui Loja Fernandès for fruitful discussions and comments on an earlier version of this paper.

\section{References}

[BMP02] M. Bordemann, A. Makhlouf, T. Petit, Déformation par quantification et rigidité des algèbres enveloppantes. Arxiv:math.RA/0211416.

[CW99] A. Cannas da Silva and A. Weinstein, Geometric models for noncommutative algebras, Berkeley Mathematics Lecture Notes, 10 (1999). American Mathematical Society, Providence, RI.

[CN82] C. Camacho and A. Lins Neto, The topology of integrable differential forms near a singularity Inst. Hautes Études Sci. Publ. Math. 55 (1982), 5-35.

[CF04] M. Crainic and R.-L. Fernandès, paper in preparation.

[CM04] M. Crainic and I. Moerdijk, Deformations of Lie brackets: cohomological aspects. Preprint Arxiv:math.DG/0403434

[C90] T. Courant, Dirac structures, Trans. A.M.S. 319 (1990), 631661.

[[DH91] J.-P. Dufour and A. Haraki, Rotationnels et structures de Poisson quadratiques, C. R. Acad. Sci. Paris Sér. I Math. 312 (1991), 137-140. 
[DW98] J.-P. Dufour and A. Wade, Formes normales de structures de Poisson ayant un 1-jet nul en un point, J. Geom. Phys. 26 (1998), 79-96.

[DZ04] J.-P. Dufour and T. Zung, Poisson structures and their normal forms, to appear in Progress in Mathematics, Birkhäuser.

[DW04] J.-P. Dufour and A. Wade, On the local structure of Dirac manifolds. Arvix math.SG/0405257

[F02] R.-L. Fernandès, Lie algebroids, holonomy and characteristic classes, Adv. Math. 170 (2002), 119-179.

[GG73] M. Golubitsky and V. Guillemin, Stable mappings and their singularities. Graduate Texts in Mathematics, Vol. 14. Springer-Verlag, New York-Heidelberg, 1973.

[HS53] G. Hochschild and J.-P. Serre, Cohomology of Lie algebras Ann. of Math. (2) 57(1953), 591-603.

[K85] J.-L. Koszul, Crochet de Schouten-Nijenhuis et cohomologie, Astérisque, hors série (1985), 257-271.

[M01] P. Monnier, Thesis Montpellier 2 (2001).

[M02] P. Monnier, A cohomology attached to a function. Arxiv:math.DG/0212045

[R02] O. Radko, A classification of topologically stable Poisson structures on a compact oriented surface, J. Symplectic Geom. 1 (2002), 523-542. 